\title{
Single-trial EEG-fMRI reveals the dynamics of cognitive function
}

\author{
Stefan Debener ${ }^{1}$, Markus Ullsperger ${ }^{2}$, Markus Siegel ${ }^{3}$ and Andreas K. Engel ${ }^{3}$ \\ ${ }^{1}$ MRC Institute of Hearing Research Southampton, Royal South Hants Hospital, Southampton, SO14 0YG, UK \\ ${ }^{2}$ Max Planck Institute for Human Cognitive and Brain Sciences, Department of Cognitive Neurology, Stephanstrasse 1A, \\ D-04103 Leipzig, Germany \\ ${ }^{3}$ Department of Neurophysiology and Pathophysiology, Center of Experimental Medicine, University Medical Center \\ Hamburg-Eppendorf, Martinistrasse 52, D-20246 Hamburg, Germany
}

\begin{abstract}
Two major non-invasive techniques in cognitive neuroscience, electroencephalography (EEG) and functional magnetic resonance imaging (fMRI), have complementary advantages with regard to their spatial and temporal resolution. Recent hardware and software developments have made it feasible to acquire EEG and fMRI data simultaneously. We emphasize the potential of simultaneous EEG and fMRI recordings to pursue new strategies in cognitive neuroimaging. Specifically, we propose that, by exploiting the combined spatiotemporal resolution of the methods, the integration of EEG and $f M R I$ recordings on a single-trial level enables the rich temporal dynamics of information processing to be characterized within spatially well-defined neural networks.
\end{abstract}

\section{Introduction}

Electroencephalography (EEG) and functional magnetic resonance imaging (fMRI) recordings provide complementary advantages with regard to the temporal and spatial resolution of brain activity. fMRI enables brain regions engaged during cognitive processes to be localized with high spatial precision; however, the blood-oxygenationlevel-dependent (BOLD) response is too slow to capture fully the rich temporal dynamics that underlie cognitive processes. By contrast, the EEG signal is directly coupled to neuronal electrical activity and has millisecond precision; however, EEG picks up a blurred spatial mixture of the underlying cortical activity and therefore provides only limited spatial resolution. Accordingly, great hope lies in the integration of EEG and fMRI to achieve both high temporal and high spatial resolution of human brain function [1-5]. Here, we propose that simultaneous EEG and fMRI recordings provide a major improvement that will advance considerably our understanding of how cognitive functions are implemented by the brain. Importantly, simultaneous EEG-fMRI recordings enable the investigation of trial-by-trial fluctuations of brain activity, which reveals important insights into the dynamics of cognitive function.

The fundamental assumption behind any integration approach is that the signals recorded in both modalities are at least partly produced by the same neural generators.

Corresponding author: Debener, S. (sdebener@soton.ac.uk).

Available online 30 October 2006.
However, a positive correlation between EEG features and the BOLD signal cannot necessarily be expected [6]. In fact, the major methodological differences of EEG and fMRI are in principle consistent with positive, negative or no correlations. When combining fMRI and EEG, it is therefore crucial to provide strong evidence that both measures refer to the same underlying substrate. After providing an overview of previously available procedures for EEG-fMRI integration, we introduce a recently established method of combining simultaneously recorded event-related EEG and fMRI on a single-trial level (Box 1). This new approach enables the study of dynamic properties of cognitive processing beyond the common focus on evoked brain responses.

\section{fMRI-informed ERP-source modeling}

Commonly, only the averaged response in the EEG signal, the event-related potential (ERP), is considered informative. Constraining the possible source locations of ERPs using neuroimaging results has a relatively long tradition [2]. In this approach, equivalent current dipoles supposed to account for the measured ERP are 'seeded' into those brain regions that are identified using neuroimaging methods such as positron emission tomography (PET) and fMRI [7-11]. Compared with the regional BOLD response, the resulting ERP-source waveforms produce more accurate information about the temporal evolution of activity in a network of cortical areas. However, this approach rests on the implicit assumption that the activity of the ERP generators leads to local hemodynamic changes that can be identified using fMRI. Cortical regions identified by fMRI do not always provide a good starting point for ERP-source modeling [3,4,12]. Each method can be blind to the activity detected by the other method under certain circumstances [13], and ERP and fMRI can differ in their sensitivity to experimental manipulations. Therefore, the corresponding neural generators might lack substantial overlap.

\section{Parametric design and EEG-fMRI covariation}

An alternative approach for integrating ERP and fMRI is based on parametric task manipulations. A range of parametrically graded experimental conditions are employed to identify cortical regions for which the BOLD response shows the same modulation across conditions as a specific 


\section{Box 1. Trial-by-trial EEG-fMRI analysis}

Recent advances in EEG hardware development have made it feasible to record multi-channel EEG data and an fMRI signal simultaneously, even at $3 \mathrm{~T}$ (Figure la of this box). EEG signals recorded inside the scanner are contaminated with two serious artifacts (Figure lb). Gradient artifacts are due to the switching of magnetic resonance (MR) gradients necessary to collect MRI data. They are relatively invariant, so gradient artifacts can be removed by subtraction procedures. The ballistocardiogram (BCG) artifact is related to the pulsatile movement of blood and the pulsatile movement of electrodes adjacent to large blood vessels. Powerful multivariate BCG-correction procedures now enable the major properties of the EEG signal to be reconstructed [20,22] (Figure lb). However, similar to EEG recordings outside the scanner, the insidescanner EEG signal corrected for MR-gradient and BCG artifacts still represents a mixture of overlapping brain and non-brain activities, such as eye blinks, spontaneous oscillations or transient, eventrelated responses. To isolate these signals, blind-source separation algorithms, such as independent component analysis (ICA), have been used successfully [22,32,33] (Figure Ic). ICA linearly decomposes EEG data corrected from MR-gradient and BCG artifacts into several components. The components can be characterized by their maximally temporally independent time courses, their condition effects and their spatial properties [35]. One or several independent components that reflect task-related EEG activity can be used to obtain single-trial EEG amplitudes. Alternatively, components that represent artifacts can be discarded and the artifact-corrected signal can be used (Figure Ic). Several studies suggest that single-trial amplitudes from selected independent components not only reflect evoked condition-related effects but also preserve event-related trialby-trial fluctuations within each condition (Figure Id). Convolution of these single-trial amplitudes with the hemodynamic response function (HRF) takes into account the temporal evolution of the fMRI BOLD response (Figure le). The result is one or several parametric regressors that can be used to predict the BOLD response that have been acquired concurrently from the same subject (Figure If). Recent work suggests that EEG-informed fMRI analysis, as described here, can help to identify brain areas that are involved in cognitive processing with more functional (and therefore spatial) specificity than the conventional analysis of fMRI alone [32,33]. EEG-informed fMRI analysis seems capable of localizing the neural generators of EEG measures, and provides a powerful way to study the functional role of dynamic trial-by-trial fluctuations of brain activity.

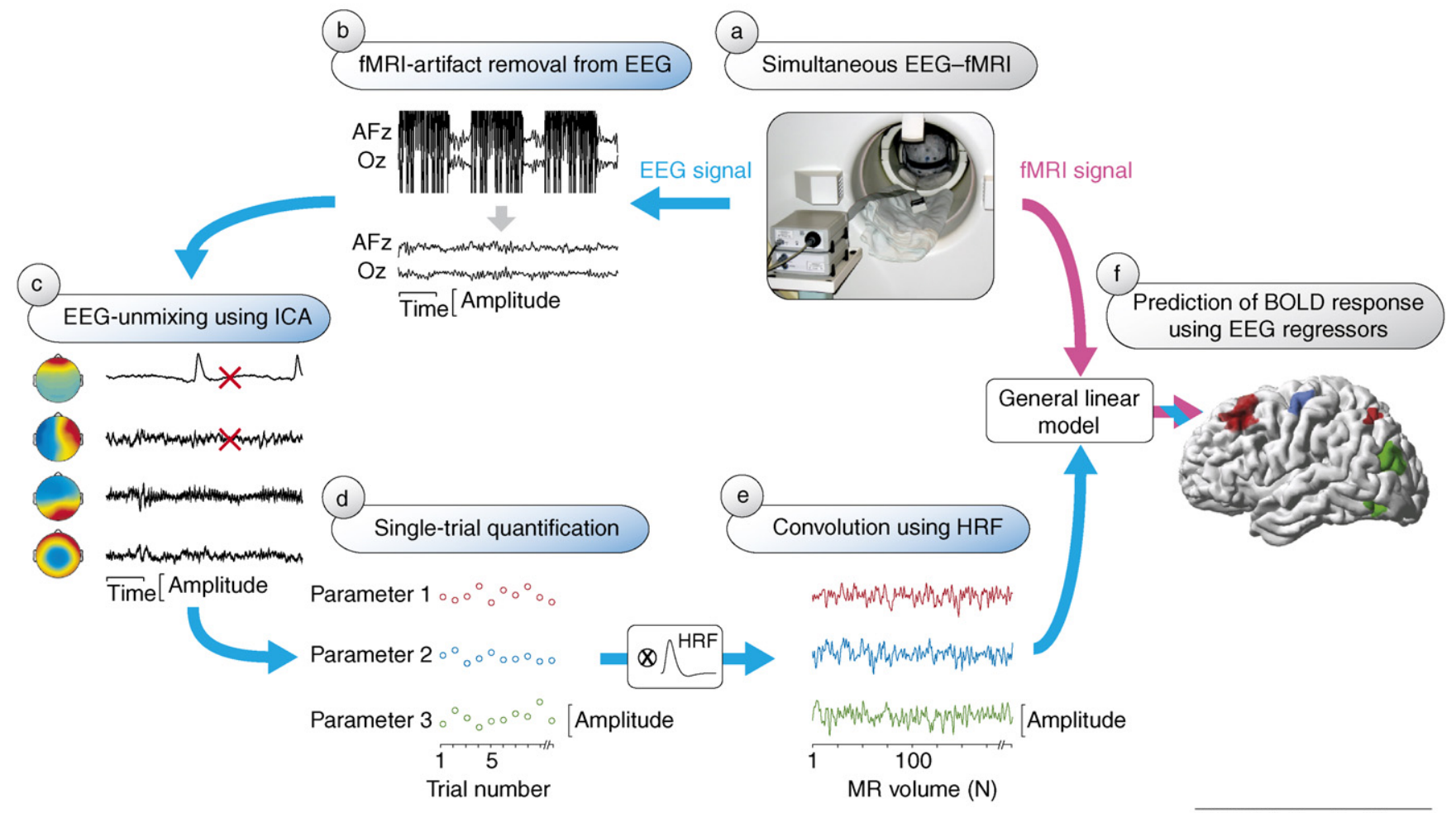

TRENDS in Cognitive Sciences

Figure I. EEG-informed fMRI analysis. EEG (blue arrows) and fMRI (pink arrow) can be recorded simultaneously (a) and, subsequently, EEG signals are corrected for fMRI artifacts. This is illustrated for two (AFz and Oz) out of a larger number of EEG channels (b). ICA applied to the continuous EEG signal returns artifact-related and brain-related component activations and maps; typical artifact-related components are marked with red crosses (c). Selected components reflecting brain activity of interest can be used to obtain a measure for each recorded trial (d). After convolution $(\otimes)$ with the hemodynamic response function (HRF), the single-trial amplitudes yield EEG regressors (e) that parametrically predict the BOLD response (f).

ERP component. Several groups have applied this method successfully to separately recorded EEG and fMRI signals and have reported systematic covariations of ERP amplitudes with regional BOLD responses [9,14-16]. The parametric-design approach helps to localize indirectly a cognitive process that is temporally defined using an ERP amplitude, without the need for simultaneous measurements of EEG and fMRI. Similar to fMRI-informed ERPsource modeling, this strategy builds on the assumption that the neural generators of BOLD and ERP responses overlap. However, the evidence of covariation across conditions provided in support of this assumption is limited. A brain region might depict the same parametric BOLD modulation as an ERP component, even though this region might not be the generator of the respective component and might exhibit a different temporal activation profile. In addition, not every experimental manipulation can be meaningfully implemented in a parametric design. Owing 
Table 1. Comparison of separate and simultaneous EEG-fMRI recording protocols

\begin{tabular}{|c|c|c|}
\hline Protocol feature & Separate & Simultaneous \\
\hline Optimal signal quality & Yes & No \\
\hline Possibility to optimize design & Yes & No \\
\hline Avoidance of order effects & No & Yes \\
\hline Identical sensory stimulation & No & Yes \\
\hline Identical subjective experience & No & Yes \\
\hline Identical behavior & No & Yes \\
\hline Direct temporal correlation of EEG and $f M R I$ signals & No & Yes \\
\hline
\end{tabular}

to the relatively small number of conditions that can be implemented during a recording, the correlation analysis often suffers from a mixture of within-subject and betweensubject effects.

\section{Virtues of simultaneous recordings}

Simultaneous EEG and fMRI recordings provide fundamental advantages over separate recording protocols (Table 1). Firstly, it is challenging, if not impossible, to provide identical sensory stimulation in two recording environments as different as EEG and fMRI laboratories. Magnetic resonance (MR) scanner noise, for instance, is partly bone-conducted and therefore difficult to simulate outside the scanner room. Furthermore, many cognitive processes, such as learning, novelty processing or object identification, are not well-suited to repeated testing because the same stimuli cannot be used twice. In addition, differences in preparation time, task experience and the recording environment itself might affect a participant's mood, vigilance, compliance and behavior, which could be reflected in different patterns of brain activity. Even in well-established cognitive experiments, the behavior of the same participant in the same task can differ substantially between EEG and fMRI recording sessions [10]. Finally, several lines of evidence suggest that ongoing fluctuations of brain activity can reflect intrinsically generated anticipatory signals that interact with, and modify, the processing of sensory events [17]. Simultaneous protocols not only guarantee identical sensory stimulation, perception and behavior, but also provide a unique way to study how these intrinsic brain states interact with event-related, extrinsic processing.

The only major concern regarding EEG-fMRI recordings is the potentially compromised EEG quality. Although it has been possible to record EEG data inside the scanner for a few years [18], more recent developments in amplifier design and artifact-correction procedures now make it much easier to obtain reasonable EEG data quality [19-22].

\section{Simultaneous EEG and fMRI of ongoing activity}

Simultaneous EEG and fMRI measurements enable covariation of both signals to be exploited on a purely temporal basis, which provides strong evidence for identifying common neural generators of fMRI and EEG. The first study to correlate both signals directly across time showed that spontaneous fluctuations of EEG alpha power $(8-12 \mathrm{~Hz})$ in a resting state covaried with simultaneously recorded regional fluctuations of the BOLD signal [23]. The pattern of correlations was consistent with structures that are thought to be directly (occipital and parietal cortex) or indirectly (thalamus) involved in the generation of EEG alpha activity. Further studies have replicated and extended this initial report [24-27]. Interestingly, these studies found BOLD activity in occipital cortex to be negatively correlated with EEG alpha activity. This finding is consistent with the idea that large-scale synchronized activity in the alpha frequency range represents an idling rhythm and corresponds to cortical inactivation [28]. This interpretation also supports the notion that simultaneous EEG-fMRI does not necessarily identify the neural generators of EEG signals, but does enable the identification of intrinsic brain states that reflect a cognitive 'default mode' expressed in both modalities $[17,27,29]$.

\section{Event-related simultaneous EEG and fMRI}

The success of EEG-fMRI studies on the resting state suggests that fluctuations of brain activity can be measured simultaneously at a temporal scale appropriate for event-related designs; that is, on a single-trial level. Box 1 illustrates processing steps that directly relate EEG and fMRI signals to each other on a single-trial level. Two initial studies reported a positive trial-by-trial correlation of the EEG-contingent negative variation with the BOLD signal in the thalamus and the posterior medial frontal cortex [30,31]. Two further studies used well-established experimental manipulations and demonstrated a direct link between event-related single-trial EEG amplitudes and cortical BOLD fMRI responses [32,33]. Eichele et al. [32] systematically manipulated the sequence of target stimuli in a target-detection (oddball) paradigm, thereby modulating the subjective predictability of target occurrence. Single-trial amplitudes of different ERP components served as predictors of the BOLD response, and several sequential spatiotemporal processing stages related to perceptual inference and pattern learning were identified. This study exemplifies a key benefit of EEGfMRI integration: on a timescale of $<0.5 \mathrm{~s}$, the EEG signal provided enough information to identify several spatially separated event-related regional activations that were differentially modulated by the experimental paradigm. Using single-trial measures of ERP components as BOLD regressors enabled corresponding brain regions to be identified using fMRI with the effective temporal resolution of the EEG.

We have recently used trial-by-trial event-related EEG amplitude variations to predict the BOLD response in a performance-monitoring task [33]. The single-trial measure of the error-related negativity correlated specifically with the BOLD signal in posterior medial frontal cortex, a key area in the monitoring of motivated behavior [34] Interestingly, the localization based on the single-trial EEG regressor was regionally more specific than conventional fMRI analysis because it avoided potential biases introduced by the subtraction logic [13] of conventional 
fMRI contrasts. Moreover, this analysis captured the parametric modulation of a more focused cognitive function, namely performance monitoring. This suggests that the trial-to-trial variability of the EEG signal provides fine-grained information that can be used to pinpoint the role of different cortical regions in a given cognitive task. Moreover, in accordance with current models of performance monitoring [34], the single-trial EEG amplitudes also predicted the slowing of subjects' reaction times in trials following response errors (Box 2). This finding is important because it demonstrates that the trial-to-trial covariation of EEG and fMRI is not due to functionally irrelevant brain activity but, by contrast, reflects behaviorally relevant neuronal activity.

It is important to consider that scalp-EEG recordings reflect a mixture of activities that are generated by an unknown number of sources. Thus, the two studies discussed $[32,33]$ and another simultaneous EEG-fMRI study [26] build on the idea of unmixing the EEG data before portions are correlated with the BOLD signal (Box 1). Unmixing methods, such as independent component analysis (ICA), can deal effectively with EEG artifacts and help to unravel spatiotemporally overlapping brain activities [35-38]. Although these EEG-fMRI studies propose different analysis strategies, they suggest that ICA helps to optimize the signal-to-noise ratio of single-trial EEG estimates used to predict the BOLD response $[26,32,33]$. The ability to estimate independent components based on a fused ERP and fMRI data space [39] might also provide important new insights, in particular when considering single-trial EEG-fMRI information from simultaneous recording protocols.

\section{Outlook and future directions}

The studies discussed in this article used single-trial quantifications related to classical ERP component quantification to integrate EEG and fMRI. However, cognitive processes are not limited to phase-locked brain responses (Box 2); they also induce non-phase-locked, spectrally specific changes of neural population activity $[28,40,41]$. Recent invasive [42-44] and non-invasive [41,45,46] studies and theoretical accounts [47] support the idea of a

\section{Box 2. Dynamic cognitive events and brain function}

Cognitive ERP and fMRI studies usually consider only event-related brain signals, which are thought to be independent from, and merely superimposed to, ongoing brain activity. According to this view, within-subject trial-by-trial fluctuations over time are treated as random noise and consequently averaged out. However, the brain can be considered a dynamic system, and a defining feature of any dynamic system is that it can undergo state changes. It is also characteristic for dynamic systems that these different states determine properties of the response to any given stimulus. Accordingly, the same stimulus can evoke different responses depending on the functional context expressed by the intrinsic system dynamics. In contrast to conventional analyses, a single-trial approach aims to take into account the functional significance of those state-dependent trialby-trial fluctuations.

By considering task-independent but region-specific decreases in brain activity, cognitive neuroimaging studies have identified a 'default mode' of brain function [17]. Activity in the default-mode network fluctuates and might define a physiological state necessary to develop and maintain an intrinsic model of anticipated events. In agreement with this view is a recent study showing that the degree of de-activation in the default-mode network is related to temporary lapses in attention [50]. Although there is a growing number of reports on systematic trial-by-trial fluctuations in EEG $[35,49]$ and fMRI [51,52], both signals also suffer from noise contributions, which are difficult to eliminate without averaging. The EEG-informed fMRIanalysis approach provides a solution to this problem (Figure la of this box). Both EEG and fMRI signals capture event-related activity and, to an unknown extent, physiological or technical noise. By using temporal correlations of EEG and fMRI (Box 1), the event-related part of brain activity captured by both measures can be identified on a single-trial level; physiological or technical noise is specific to each recording technique and, therefore, can be assumed not to contribute to this correlation (Figure lb). A second virtue of this approach is that it preserves the dynamic aspects of cognitive function. For example, performance monitoring refers to a process that detects whether action outcomes deviate from action goals and initiates adjustments that optimize future behavior whenever the outcome is worse than intended. Performance monitoring is modulated by state variables, such as motivation, and it seems conceivable that performance monitoring fluctuates owing to interactions with the default mode of brain activity [50]. Single-trial EEG amplitudes might be related to this fluctuation and can be used to predict the subjects' reaction times in performance tasks [33] (Figure Ic).

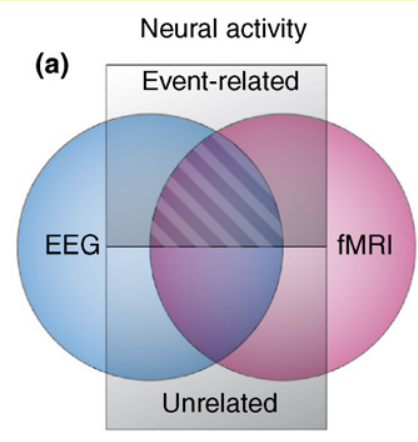

(b)

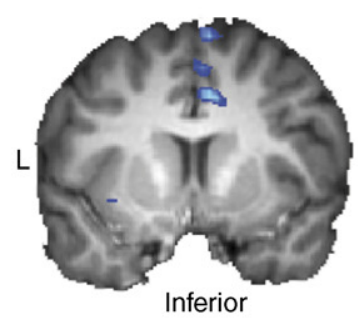

(c)

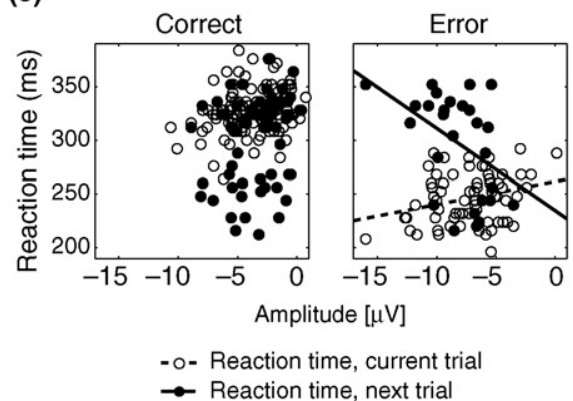

Figure I. EEG-informed fMRI analysis reveals dynamic properties of cognitive processing. (a) The virtues of the temporal correlation approach. Event-related EEG-fMRI coupling, if present, is thought to represent a fraction of the variance of each measure (hatched area). (b) Event-related trial-by-trial EEG fluctuations can be used to identify medial frontal brain areas that are involved in performance monitoring in a single subject. (c) The claim that medial frontal brain areas are involved in performance monitoring is supported by behavioral results. Single-trial amplitudes of the error-related negativity, a component reflecting performance monitoring, were used to predict the participants' reaction times in the same, and the subsequent, trial. Only in response error trials (right) did higher single-trial EEG amplitudes predict longer reaction times on the next trial, an effect known as post-error slowing. Figure Ic reproduced, with permission, from Ref. [33], (C) (2005) by the Society for Neuroscience. 


\section{Box 3. Questions for future research}

- What is the spectral signature of correlations between BOLD fMRI and EEG? Is there a homogenous relationship over distinct cortical areas?

- What is the relationship between ongoing and event-related EEG oscillations regarding correlations with the BOLD signal?

- Under which sensory and cognitive circumstances do EEG signals fail to correlate systematically with the BOLD signal?

- ERP components are often characterized by more than one ICA component. What is the relationship between independent components and conventional ERP components regarding the correlation with the BOLD signal?

- Does the cognitive default mode of brain activity, as revealed by BOLD fMRI, account for event-related EEG trial-by-trial fluctuations?

- Can 'true data fusion' techniques, such as the joint ICA of EEG and $f M R I$ data, help to describe EEG and fMRI signals that are directly coupled to each other?

spectrally specific correlation between neural population activity and BOLD fMRI. In particular, high-frequency activity in the gamma band seems to be positively correlated with the BOLD response, whereas alpha band activity seems to be negatively correlated. An important related question concerns the interaction between ongoing and event-related brain activity. For instance, it is conceivable that neuronal assemblies that give rise to evoked EEG signals have a modulating influence on ongoing activity, and vice versa. Moreover, it has been reported that eventrelated EEG responses are due to a partial phase resetting of, and thus not independent from, ongoing activity. Therefore, ERPs originating from partial phase resetting $[35,48,49]$ might not induce major changes in local brain metabolism, and thus might not correlate with the BOLD signal. By contrast, additive event-related responses due to a larger neural assembly should be correlated with the local event-related fMRI BOLD signal [48]. Future studies investigating these issues should profit from simultaneous EEG-fMRI designs and single-trial EEG responses, characterized in the time-frequency domain as regressors for the BOLD fMRI signal (Box 3).

The recent advent of simultaneous EEG-fMRI recording protocols provides a key non-invasive technique for directly relating electromagnetic and hemodynamic signals. Simultaneous recordings provide a unique way to investigate event-related trial-by-trial correlations between these measures. Such direct correlations provide valuable evidence; we propose that they provide the best means to localize non-invasively those structures that underlie temporally well-resolved EEG responses. Furthermore, they enable the identification of systematic trial-by-trial fluctuations of brain activity, thereby avoiding the questionable assumption of a clear-cut distinction between ongoing and additive event-related brain responses. Accordingly, we claim that single-trial EEGinformed fMRI analysis provides a powerful tool for characterizing the neural dynamics of cognitive processes and offers previously unattained effective spatiotemporal resolution.

\section{Acknowledgements}

We are grateful to C. Kranczioch and T. Eichele for providing helpful comments on the manuscript.

\section{References}

1 Babiloni, F. et al. (2004) Multimodal integration of EEG, MEG and fMRI data for the solution of the neuroimage puzzle. Magn. Reson. Imaging 22, 1471-1476

2 Heinze, H.J. et al. (1994) Combined spatial and temporal imaging of brain activity during visual selective attention in humans. Nature 372 543-546

3 Dale, A.M. et al. (2000) Dynamic statistical parametric mapping: combining fMRI and MEG for high-resolution imaging of cortical activity. Neuron $26,55-67$

4 Dale, A.M. and Halgren, E. (2001) Spatiotemporal mapping of brain activity by integration of multiple imaging modalities. Curr. Opin. Neurobiol. 11, 202-208

5 Horwitz, B. and Poeppel, D. (2002) How can EEG/MEG and fMRI/PET data be combined? Hum. Brain Mapp. 17, 1-3

6 Nunez, P.L. and Silberstein, R.B. (2000) On the relationship of synaptic activity to macroscopic measurements: does co-registration of EEG with fMRI make sense? Brain Topogr. 13, 79-96

7 Bledowski, C. et al. (2004) Localizing P300 generators in visual target and distractor processing: a combined event-related potential and functional magnetic resonance imaging study. J. Neurosci. 24, 9353-9360

8 Gotman, J. et al. (2004) Combining EEG and fMRI in epilepsy: methodological challenges and clinical results. J. Clin. Neurophysiol. $21,229-240$

9 Schicke, T. et al. (2006) Tight covariation of BOLD signal changes and slow ERPs in the parietal cortex in a parametric spatial imagery task with haptic acquisition. Eur. J. Neurosci. 23, 1910-1918

10 Ullsperger, M. and von Cramon, D.Y. (2001) Subprocesses of performance monitoring: a dissociation of error processing and response competition revealed by event-related fMRI and ERPs. Neuroimage 14, 1387-1401

11 Brass, M. et al. (2005) Who comes first? The role of the prefrontal and parietal cortex in cognitive control. J. Cogn. Neurosci. 17, 1367-1375

12 Ahlfors, S.P. et al. (1999) Spatiotemporal activity of a cortical network for processing visual motion revealed by MEG and fMRI. $J$. Neurophysiol. 82, 2545-2555

13 Friston, K.J. et al. (1998) Event-related fMRI: characterizing differential responses. Neuroimage 7, 30-40

14 Horovitz, S.G. et al. (2004) Parametric design and correlational analyses help integrating fMRI and electrophysiological data during face processing. Neuroimage 22, 1587-1595

15 Horovitz, S.G. et al. (2002) Correlations and dissociations between BOLD signal and P300 amplitude in an auditory, oddball task: a parametric approach to combining fMRI and ERP. Magn. Reson. Imaging 20,319-325

16 Mulert, C. et al. (2005) Sound level dependence of the primary auditory cortex: Simultaneous measurement with 61-channel EEG and fMRI Neuroimage 28, 49-58

17 Raichle, M.E. and Gusnard, D.A. (2005) Intrinsic brain activity sets the stage for expression of motivated behavior. J. Comp. Neurol. 493, $167-176$

18 Kruggel, F. et al. (2000) Recording of the event-related potentials during functional MRI at 3.0 Tesla field strength. Magn. Reson. Med. 44, 277-282

19 Iannetti, G.D. et al. (2005) Simultaneous recording of laser-evoked brain potentials and continuous, high-field functional magnetic resonance imaging in humans. Neuroimage 28, 708-719

20 Niazy, R.K. et al. (2005) Removal of FMRI environment artifacts from EEG data using optimal basis sets. Neuroimage 28, 720-737

21 Sammer, G. et al. (2005) Acquisition of typical EEG waveforms during fMRI: SSVEP, LRP, and frontal theta. Neuroimage 24, 1012-1024

22 Debener, S. et al. Improved quality of auditory event-related potentials recorded simultaneously with 3-T fMRI: removal of the ballistocardigoram artefact. Neuroimage (in press)

23 Goldman, R.I. et al. (2002) Simultaneous EEG and fMRI of the alpha rhythm. Neuroreport 13, 2487-2492

24 Moosmann, M. et al. (2003) Correlates of alpha rhythm in functional magnetic resonance imaging and near infrared spectroscopy. Neuroimage 20, 145-158

25 Martinez-Montes, E. et al. (2004) Concurrent EEG/fMRI analysis by multiway Partial Least Squares. Neuroimage 22, 1023-1034

26 Feige, B. et al. (2005) Cortical and subcortical correlates of electroencephalographic alpha rhythm modulation. J. Neurophysiol. 93, 2864-2872 
27 Laufs, H. et al. (2003) EEG-correlated fMRI of human alpha activity. Neuroimage 19, 1463-1476

28 Pfurtscheller, G. and Lopes da Silva, F.H. (1999) Event-related EEG/ MEG synchronization and desynchronization: basic principles. Clin. Neurophysiol. 110, 1842-1857

29 Laufs, H. et al. (2003) Electroencephalographic signatures of attentional and cognitive default modes in spontaneous brain activity fluctuations at rest. Proc. Natl. Acad. Sci. U. S. A. 100, 11053-11058

30 Hinterberger, T. et al. (2005) Neuronal mechanisms underlying control of a brain-computer interface. Eur. J. Neurosci. 21, 3169-3181

31 Nagai, Y. et al. (2004) Brain activity relating to the contingent negative variation: an fMRI investigation. Neuroimage 21, 1232-1241

32 Eichele, T. et al. (2005) Assessing the spatiotemporal evolution of neuronal activation with single-trial event-related potentials and functional MRI. Proc. Natl. Acad. Sci. U. S. A. 102, 17798-17803

33 Debener, S. et al. (2005) Trial-by-trial coupling of concurrent electroencephalogram and functional magnetic resonance imaging identifies the dynamics of performance monitoring. J. Neurosci. 25, 11730-11737

34 Ridderinkhof, K.R. et al. (2004) The role of the medial frontal cortex in cognitive control. Science 306, 443-447

35 Makeig, S. et al. (2004) Mining event-related brain dynamics. Trends Cogn. Sci. 8, 204-210

36 Brown, G.D. et al. (2001) Independent component analysis at the neural cocktail party. Trends Neurosci. 24, 54-63

37 Delorme, A. and Makeig, S. (2004) EEGLAB: an open source toolbox for analysis of single-trial EEG dynamics including independent component analysis. J. Neurosci. Methods 134, 9-21

38 Debener, S. et al. (2005) What is novel in the novelty oddball paradigm? Functional significance of the novelty P3 event-related potential as revealed by independent component analysis. Cognitive Brain Research 22, 309-321
39 Calhoun, V.D. et al. (2006) Neuronal chronometry of target detection: fusion of hemodynamic and event-related potential data. Neuroimage $30,544-553$

40 Makeig, S. et al. (2004) Electroencephalographic brain dynamics following manually responded visual targets. PLoS Biol. 2, 747-762

41 Siegel, M. et al. (2006) High-frequency activity in human visual cortex is modulated by visual motion strength. Cerebral Cortex, DOI: 10.1093/ cercor/bhk025 (http://cercor.oxfordjournals.org/)

42 Logothetis, N.K. et al. (2001) Neurophysiological investigation of the basis of the fMRI signal. Nature 412, 150-157

43 Mukamel, R. et al. (2005) Coupling between neuronal firing, field potentials, and fMR1 in human auditory cortex. Science 309, 951-954

44 Niessing, J. et al. (2005) Hemodynamic signals correlate tightly with synchronized gamma oscillations. Science 309, 948-951

45 Brookes, M.J. et al. (2005) GLM-beamformer method demonstrates stationary field, alpha ERD and gamma ERS co-localisation with fMRI BOLD response in visual cortex. Neuroimage 26, 302-308

46 Hall, S.D. et al. (2005) The missing link: analogous human and primate cortical gamma oscillations. Neuroimage 26, 13-17

47 Kilner, J.M. et al. (2005) Hemodynamic correlates of EEG: a heuristic. Neuroimage 28, 280-286

48 Fell, J. et al. (2004) Neural bases of cognitive ERPs: more than phase reset. J. Cogn. Neurosci. 16, 1595-1604

49 Makeig, S. et al. (2002) Dynamic brain sources of visual evoked responses. Science 295, 690-694

50 Weissman, D.H. et al. (2006) The neural bases of momentary lapses in attention. Nat. Neurosci. 9, 971-978

51 Fox, M.D. et al. (2006) Coherent spontaneous activity accounts for trial-to-trial variability in human evoked brain responses. Nat. Neurosci. 9, 23-25

52 Fox, M.D. et al. (2005) The human brain is intrinsically organized into dynamic, anticorrelated functional networks. Proc. Natl. Acad. Sci. U. S. A. $102,9673-9678$

\section{Articles of interest in other Trends and Current Opinion journals}

Neurons in motion: same principles for different shapes?

Oscar MarÚn, Miguel Valdeolmillos and Fernando Moya

Trends in Neurosciences DOI:10.1016/j.tins.2006.10.001

Neurocognitive aging: prior memories hinder new hippocampal encoding

lain A. Wilson, Michela Gallagher, Howard Eichenbaum and Heikki Tanila Trends in Neurosciences DOI:10.1016/j.tins.2006.10.002

Targeting adenosine A2A receptors in Parkinson's disease

Michael A. Schwarzschild, Luigi Agnati, Kjell Fuxe, Jiang-Fan Chen and Micaela Morelli Trends in Neurosciences DOI:10.1016/j.tins.2006.09.004

Lipoprotein receptors in Alzheimer's disease

Olav M. Andersen and Thomas E. Willnow

Trends in Neurosciences DOI:10.1016/j.tins.2006.09.002

Exciting inhibition in psychostimulant addiction

Peter W. Kalivas and Xiu-Ti Hu

Trends in Neurosciences DOI:10.1016/j.tins.2006.08.008

Assessing sensory function in locomotor systems using neuro-mechanical simulations

Keir Pearson, Örjan Ekeberg and Ansgar Büschges

Trends in Neurosciences DOI:10.1016/j.tins.2006.08.007

Did brain-specific genes evolve faster in humans than in chimpanzees?

Peng Shi, Margaret A. Bakewell and Jianzhi Zhang

Trends in Genetics DOI: 10.1016/j.tig.2006.09.001

Understanding the molecular causes of Parkinson's disease

A. Wood-Kaczmar, S. Gandhi and N.W. Wood

Trends in Molecular Medicine DOI:10.1016/j.molmed.2006.09.007

The role of acetylcholine in learning and memory

Michael E. Hasselmo

Current Opinion in Neurobiology DOI:10.1016/j.conb.2006.09.002

Essential and expendable features of the circadian timekeeping mechanism

Paul E. Hardin

Current Opinion in Neurobiology DOI:10.1016/j.conb.2006.09.001

Scanning the human genome for signals of selection

Gil McVean and Chris CA Spencer

Current Opinion in Genetics and Development DOI:10.1016/j.gde.2006.09.004 